\title{
Mechanistic insights into copper-catalyzed carboxylations
}

\author{
Marc F. Obst, ${ }^{\dagger}$ Ashot Gevorgyan, ${ }^{\ddagger}$ Annette Bayer, ${ }^{*, \ddagger}$ and Kathrin H. Hopmann ${ }^{*,+}$ \\ †Hylleraas Center for Quantum Molecular Sciences, Department of Chemistry, UiT The Arctic University of \\ Norway, kathrin.hopmann@uit.no \\ ‡Department of Chemistry, UiT The Arctic University of Norway, annette.bayer@uit.no
}

\section{Abstract}

The copper-NHC-catalyzed carboxylation of organoboranes with $\mathrm{CO}_{2}$ was investigated using computational and experimental methods. The DFT and DLPNO-CCSD(T) results indicate that nonbenzylic substrates are converted via an inner sphere carboxylation of an organocopper intermediate, whereas benzylic substrates may simultaneously proceed along both inner and outer sphere $\mathrm{CO}_{2}$ insertion pathways. Interestingly, the computations predict that two conceptually different carboxylation mechanisms are possible for benzylic organoboranes, one being coppercatalyzed and one being mediated by the reaction additive CsF. Our experimental evaluation of the computed reactions confirms that carboxylation of non-benzylic substrates requires coppercatalysis, whereas benzylic substrates can be carboxylated with and without copper.

\section{Introduction}

Carbon dioxide has significant potential as a carbon source for chemical synthesis, because it is plentiful, readily available, and cheap. ${ }^{1,2-7}$ In this function, $\mathrm{CO}_{2}$ could help replace fossil carbon sources, such as crude oil or natural gas, thus reducing the dependency on these finite resources. A challenge in this approach is the relatively high chemical inertness of $\mathrm{CO}_{2}$, resulting in the necessity for catalysts or reactive co-substrates for many reactions involving $\mathrm{CO}_{2}$. Nevertheless, $\mathrm{CO}_{2}$-based synthesis pathways for a variety of products have been developed, which can be classified into three categories: i) reactions selectively reducing $\mathrm{CO}_{2}$ to formic acid, $\mathrm{CO}$, methanol, or lower alkanes, ii) reactions fixating $\mathrm{CO}_{2}$ through carbon-carbon (C-C) bond formation to form e.g. 
carboxylic acids, and iii) reactions fixating $\mathrm{CO}_{2}$ through carbon-heteroatom (C-X) bond formation, leading to cyclic carbonates or carbamates. ${ }^{6-8}$

A $\mathrm{Cu}(\mathrm{I})$-based system able to fixate $\mathrm{CO}_{2}$ through $\mathrm{C}-\mathrm{C}$ bond formation was published independently by $\mathrm{Hou}^{9}$ and Sawamura ${ }^{10}$ in 2011, and improved upon by Skrydstrup, Nielsen, and coworkers $^{11}$ in 2017 (Scheme 1). The reaction protocol involves two steps: first, a hydroboration with the 9-borabicyclo[3.3.1]nonane dimer $(9-\mathrm{BBN})_{2}$ and second, a 1,10-phenanthrolineCu(I)- or $\operatorname{IPrCu}(\mathrm{I})$-catalyzed (IPr = 1,3-bis(2,6-diisopropylphenyl)imidazol-2-ylidene) carboxylation of the in situ formed organoborane with $\mathrm{CO}_{2}$ in the presence of a base. Skrydstrup and coworkers employed the milder base CSF instead of the strong alkoxides used by Sawamura or Hou, expanding the scope of substrates to cyclic olefins, stilbenes, $\beta$-substituted styrenes and terminal acetylenes. ${ }^{9-11}$
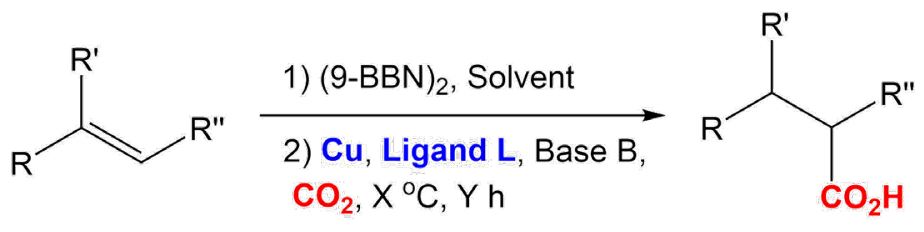

Scheme 1. Cu-catalyzed carboxylations reported by Hou $(X=70, Y=24, \mathrm{Cu}=\mathrm{CuCl}, \mathrm{L}=\mathrm{IPr}, \mathrm{B}=\mathrm{MeOLi}, \mathrm{R}$ " $=\mathrm{H})$, Sawamura $\left(X=100, Y=12, \mathrm{Cu}=\mathrm{CuOAc}, \mathrm{L}=1,10\right.$-phen, $\left.\mathrm{B}=\mathrm{KO}^{t} \mathrm{Bu}, \mathrm{R}^{\prime \prime}=\mathrm{H}\right)$, and Skrydstrup $(X=120, Y=16, \mathrm{Cu}=\mathrm{Cul}, \mathrm{L}=\operatorname{IPr}, \mathrm{B}=$ CsF, R" = alkyl). ${ }^{9-11}$

We here set out to investigate the mechanistic details of the Cu-NHC-catalyzed carboxylation of in situ formed organoboranes. ${ }^{11}$ To achieve this goal, we employed computational methods, namely Density Functional Theory (DFT) and Domain-based Local Pair Natural Orbital Coupled Cluster (DLPNO-CCSD(T)). We were particularly interested to establish if the $\mathrm{CO}_{2}$ molecule experiences activation from the copper center during $\mathrm{C}-\mathrm{C}$ bond formation (implying an inner sphere mechanism), or if an outer sphere path with a free $\mathrm{CO}_{2}$ molecule is preferred, as recently proposed by us for Rh-catalyzed hydrocarboxylation. ${ }^{12}$ Further, it can be noted that the Cu-catalyzed reaction reported by Skrydstrup, Nielsen, and coworkers occurs in the presence of three equivalents of $\mathrm{CsF}^{11}$ and we thus paid particular attention to the role of CsF in the reaction mechanism, because we recently showed that it may promote carboxylations. ${ }^{13}$ Different types of substrates were investigated, involving both a benzylic and a non-benzylic organoborane, which here are shown to have different mechanistic preferences. Our experiments support the computational predictions. 


\section{Computational details}

Computational Model: All calculations were performed on the full molecular systems without any truncations or symmetry constraints. The two in situ formed organoboranes sub1 and sub2 (Figure 1) derived from cyclohexene $\mathbf{R} \mathbf{1}$ and trans- $\beta$-methylstyrene $\mathbf{R 2}$, respectively, were used to investigate the reaction mechanism. These substrates were chosen because they previously showed high yields in experiments (sub1: 94\%, sub2: $89 \%)^{11}$ while having different electronic properties. For steps where CsF enters or leaves, the reference structure for CsF was modelled as a dimer, $\mathrm{Cs}_{2} \mathrm{~F}_{2}$. However, as the exact nature of the $\mathrm{CsF}$ reference state under experimental conditions (dioxane solvent, $120^{\circ} \mathrm{C}$ ) is not known, we also computed the oversll barriers with an alternative tetrameric reference state $\left(\mathrm{Cs}_{4} \mathrm{~F}_{4}\right)$ for comparison (Table S1, Scheme S1).

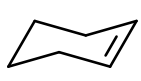

R1

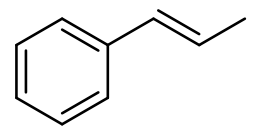

R2

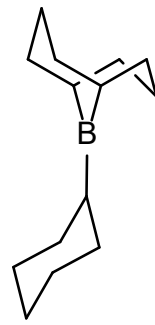

sub1

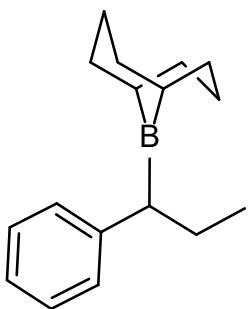

sub2

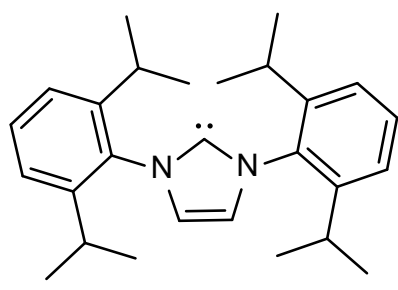

IPr

Figure 1. The two investigated organoboranes sub1 (derived from cyclohexene R1) and sub2 (derived from trans- $6 \beta$ methylstyrene $\mathbf{R} \mathbf{2}$ ) as well as the used NHC ligand IPr.

Computational Methods: Geometries were optimized using the long-range corrected $\omega \mathrm{B} 97 \mathrm{XD}$ functional. ${ }^{14}$ This functional was chosen based on good results in recent benchmarks. ${ }^{15,16}$ Additional DFT calculations were performed using the $\mathrm{PBE}^{17,18}$ and B3LYP ${ }^{19}$ functionals with the GD3BJ 20 dispersion correction to assess the robustness of the computed results (details are given in the Supporting Information, SI). The employed software was Gaussian16 B.01 for all DFT calculations. ${ }^{21}$ For optimizations, the basis set BS1 was used, which consists of the SDD basis for Cu and Cs, as well as the $6-31+G^{*}$ basis for all other elements. DFT single-point (SP) energies were calculated at the BS2 level, using the same DFT functional as used in optimizations. BS2 comprises the SDD basis for $\mathrm{Cu}$ and the def2-TZVP basis for all other elements. Both BS1 and BS2 employed the SDD ECPs for $\mathrm{Cu}$ and Cs. Solvation effects were included in optimizations and SP calculations through the use of an IEFPCM model of 1,4-dioxane.

In order to obtain accurate electronic energies beyond DFT, we employed DLPNO-CCSD(T). ${ }^{22,23}$ 
This method is reported to have a comparable accuracy to $\operatorname{CCSD}(T)$, while scaling considerably better, allowing the treatment of large molecules with high accuracy. ${ }^{24-26}$ The DLPNO-CCSD(T) SP energies were calculated using the $\omega$ B97XD-based geometries obtained as described above. The DLPNO-CCSD(T) method was used with the scalar-relativistic ZORA operator and basis set BS3, consisting of the SARC-ZORA-TZVPP basis for Cs and the minimally augmented ma-ZORA-def2TZVPP basis for all other elements. These calculations were accelerated by using the RIJCOSX approximation $^{27}$ employing the auxiliary basis sets def2-TZVP/C and SARC/J. The employed software for these calculations was ORCA 4.1.2. ${ }^{28}$ Final DLPNO-CCSD(T) standard state Gibbs free energies $\left(\Delta G_{C C S D(T)}\right)$ were obtained by applying the $\omega$ B97XD-based thermal, entropic, and solvation corrections at $393.15 \mathrm{~K}\left(120^{\circ} \mathrm{C}\right)$ and the BS1 level to the SP DLPNO-CCSD(T) electronic energies. Unless explicitly stated otherwise, the $\Delta G_{C C S D(T)}$ energies are used for the discussion.

$$
\Delta G_{C C S D(T)}=\Delta G_{(\omega B 97 \times D, P C M, B S 1)}-\Delta E_{(\omega B 97 \times D, V a c, B S 1)}+\Delta E_{(C C S D(T), V a c, B S 3)}
$$

Final DFT standard state Gibbs free energies were obtained similarly by applying the BS1 thermal and entropic corrections (393.15 K) to the SP DFT energies calculated at the BS2 level:

$$
\Delta G_{D F T}=\Delta G_{(D F T, P C M, B S 1)}-\Delta E_{(D F T, P C M, B S 1)}+\Delta E_{(D F T, P C M, B S 2)}
$$

All energies correspond to a $1 \mathrm{~atm}$ standard state.

Experimental Methods: Commercially available starting materials, reagents, catalysts and anhydrous and degassed solvents were used without further purification. Thin layer chromatography was carried out using Merck TLC Silica gel $60 \mathrm{~F}_{254}$ and visualized by shortwavelength ultraviolet light or by treatment with potassium permanganate $\left(\mathrm{KMnO}_{4}\right)$ stain. ${ }^{1} \mathrm{H}$, and ${ }^{13} \mathrm{C}$ NMR spectra were recorded on a Bruker Avance $400 \mathrm{MHz}$ at $20{ }^{\circ} \mathrm{C}$. All ${ }^{1} \mathrm{H}$ NMR spectra are reported in parts per million (ppm) downfield of TMS and were measured relative to the signals for $\mathrm{CHCl}_{3}$ (7.26 ppm). All ${ }^{13} \mathrm{C} \mathrm{NMR} \mathrm{spectra} \mathrm{were} \mathrm{reported} \mathrm{in} \mathrm{ppm} \mathrm{relative} \mathrm{to} \mathrm{residual} \mathrm{CDCl}_{3}(77.20 \mathrm{ppm})$ and were obtained with ${ }^{1} \mathrm{H}$ decoupling. Coupling constants, J, are reported in $\mathrm{Hertz}(\mathrm{Hz})$. Highresolution mass spectra (HRMS) were recorded from methanol solutions on an LTQ Orbitrap XL 
(Thermo Scientific) in negative electrospray ionization (ESI) mode. Melting points were measured using Stuart SMP50 automatic melting point detector.

General procedure for $\mathrm{Cu}$-catalyzed/Cu-free hydrocarboxylation. Inside a glove box, a $45 \mathrm{~mL}$ pressure tube was charged with corresponding olefin $(1.7 \mathrm{mmol}),(9-\mathrm{BBN})_{2}$ (1 equiv.) and dry dioxane (4 mL (Cu-catalyzed), $6 \mathrm{~mL}$ (Cu-free)). The flask was closed with suitable cap, removed from the glove box and heated to $65^{\circ} \mathrm{C}$ for $16 \mathrm{~h}$. Afterwards, the pressure tube was transferred back to the glove box. To the reaction mixture at $20^{\circ} \mathrm{C}$ was added CsF ( 3 equiv.) and, in case of Cu-catalyzed reactions, a solution of catalyst (the mixture of $\mathrm{Cul}(5 \mathrm{~mol} \%), \mathrm{PrHCl}(6 \mathrm{~mol} \%)$ and $\mathrm{NaOtBu}(6 \mathrm{~mol} \%)$ in dry dioxane $(3 \mathrm{~mL})$ stirred at $20^{\circ} \mathrm{C}$ for $30 \mathrm{~min}$ before use). The pressure tube was closed with the cap and removed from the glove box. Afterwards, $\mathrm{CO}_{2}(120 \mathrm{~mL})$ was added via a syringe, followed by stirring the reaction mixture at $120{ }^{\circ} \mathrm{C}$ for $16 \mathrm{~h}$. Next, the reaction mixture was diluted with 30 $\mathrm{mL} \mathrm{Et}{ }_{2} \mathrm{O}$ and transferred into a $500 \mathrm{~mL}$ separating funnel. The resulting mixture was extracted with $30 \mathrm{~mL}$ saturated basic $\left(\mathrm{NaHCO}_{3}\right.$ ) solution (3 times). Resulting basic solution was washed with $15 \mathrm{~mL}$ $\mathrm{Et}_{2} \mathrm{O}$ (once), acidified (50-55 $\mathrm{mL} 6 \mathrm{M} \mathrm{HCl}$ ) and extracted with $30 \mathrm{~mL} \mathrm{Et}_{2} \mathrm{O}$ (3 times). The resulting solution of $\mathrm{Et}_{2} \mathrm{O}$ was distilled to dryness to give the corresponding acid.

\section{Results and Discussion}

Cu(I)-catalyzed conversion of sub1: We initiated our investigation of the copper-catalyzed carboxylation of organoboranes from the mechanism proposed by Skrydstrup, Nielsen and coworkers. ${ }^{11}$ Their schematic proposal suggests a transmetalation of I-Cu-IPr with CsF to form the active species F-Cu-IPr, which reacts with the organoborane to give a Cu-alkyl intermediate, followed by insertion of $\mathrm{CO}_{2}$. Our computational results indicate that such a mechanism is feasible for the cyclohexene-derived organoborane sub1, although our data suggests some modifications to the original proposal, with the obtained mechanism shown in Scheme 2. We will refer to this mechanism as $\mathbf{A}$, with the computed Gibbs energy profile for sub1 shown in Figure 2. 


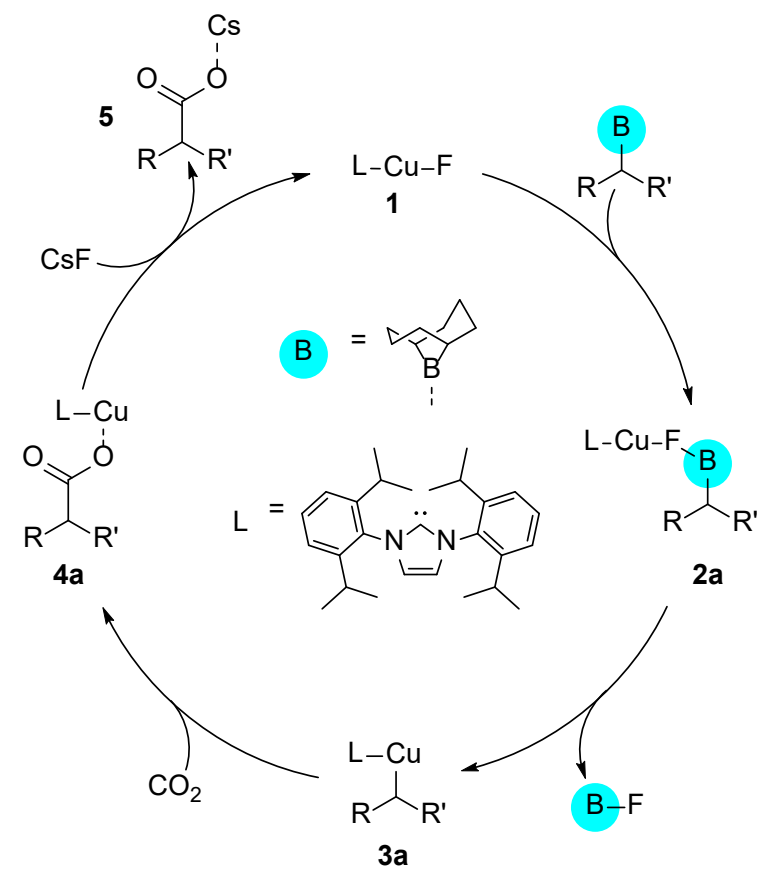

The computed pathway A starts with a nucleophilic attack of the fluoride ion of the active species (F-Cu-IPr) at the boron atom of the substrate. The corresponding transition state $\left(\mathrm{TS}_{1-2 \mathrm{a}}\right)$ has a barrier of $16.8 \mathrm{kcal} / \mathrm{mol}$ relative to 1 and is characterized by a partial transfer of the fluoride from copper to boron. At the intermediate $\mathbf{2 a}$, the fluoride is bridging between boron and the metal center. The energy of this intermediate is $7.2 \mathrm{kcal} / \mathrm{mol}$. In the following step, a concerted rearrangement leads to the formation of the Cu-alkyl intermediate $\mathbf{3 a}$, concomitant with release of (9-BBN)-F. This step has a barrier of $22.8 \mathrm{kcal} / \mathrm{mol}$ and can be viewed as a transmetalation, with the cyclic TS (TS 2 a-3a, Figure 3) composed of copper, boron and the reactive carbon. When this TS is reached, the fluoride is already fully transferred to boron. A separate TS for fluoride transfer could not be identified. The formed Cu-alkyl intermediate 3 a has a relative energy of $0.4 \mathrm{kcal} / \mathrm{mol}$, making it relatively stable. $3 a$ can then insert a $\mathrm{CO}_{2}$ molecule via $\mathbf{T S}_{\mathbf{3 a}-4 \mathbf{4}}$. Several TSs were identified for this step, which can be classified as inner or outer sphere, depending on the presence of $\mathrm{Cu}-\mathrm{CO}_{2}$ interactions at the transition state. With a computed barrier of $31.9 \mathrm{kcal} / \mathrm{mol}$, the inner sphere TS is clearly preferred for sub1, compared to the outer sphere TS with a barrier of $39.6 \mathrm{kcal} / \mathrm{mol}$ (Figure 4). The energies obtained with different DFT functionals ( $\omega B 97 X D$, PBE-D3BJ, B3LYP-D3BJ) provide the same clear preference for an inner sphere attack (SI, Table S2). This result is in line with the 


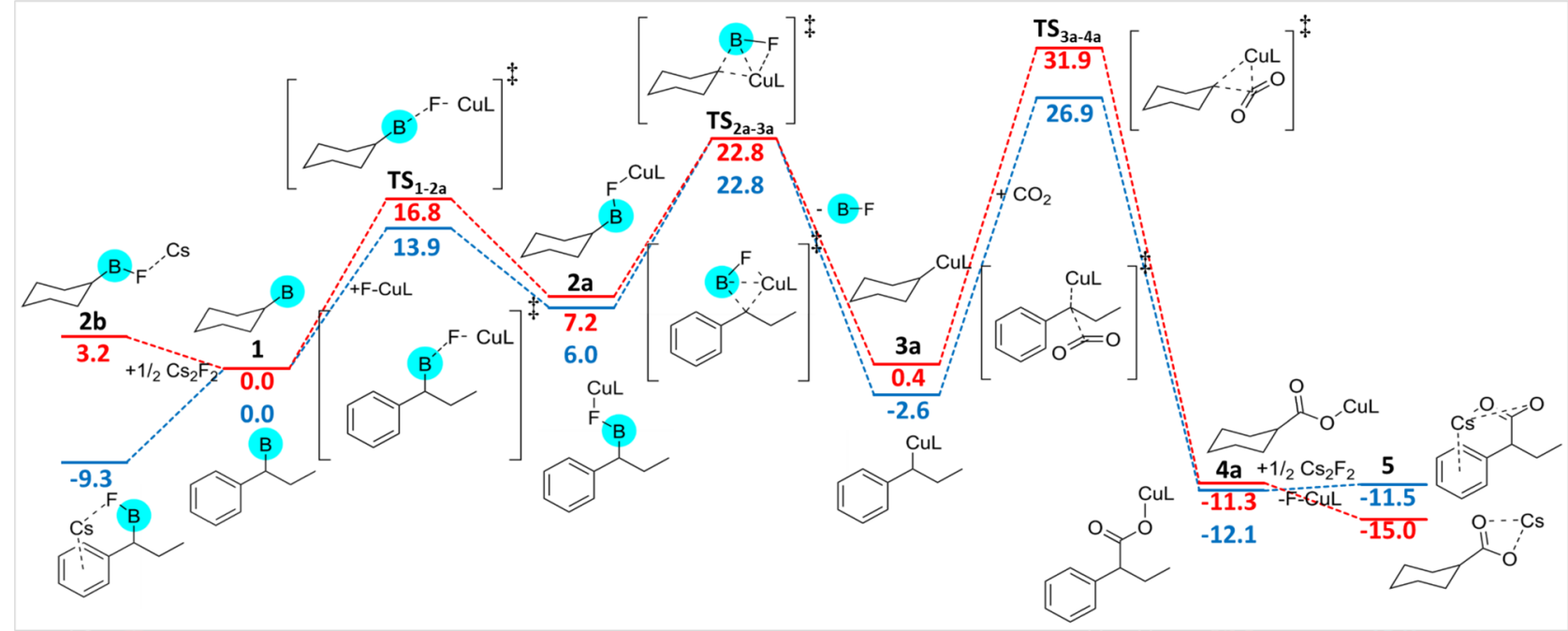

Figure 2. Computed Gibbs free energy profile (kcal/mol) of the $\mathrm{Cu}(\mathrm{l})$-catalyzed path A for sub1 (red) and sub2 (blue). $B=9-B B N, L=I P r$.

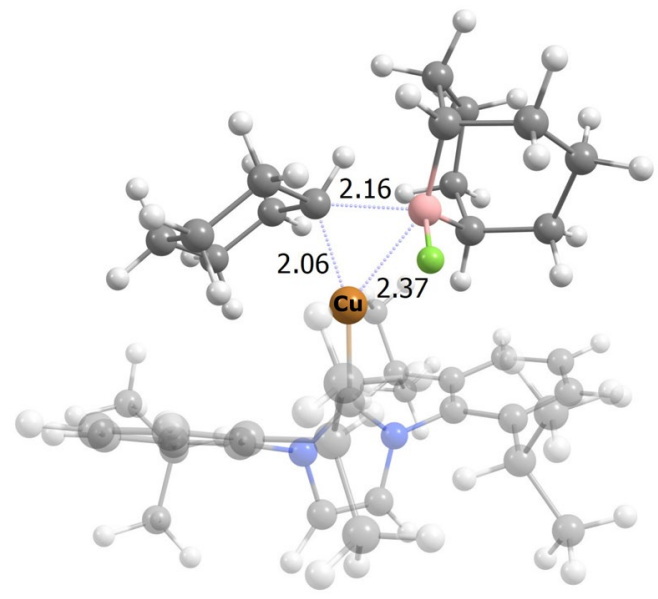

Figure 3. Optimized geometry of TS2a-3a for sub1. Distances are in $\AA$. The NHC ligand (IPr) is transparent for clarity. Color code: $\mathrm{C}=$ grey, $\mathrm{H}=$ white, $\mathrm{N}=$ blue, $\mathrm{B}=$ pink, $\mathrm{F}=$ green, $\mathrm{O}=$ red.

inner sphere mechanism predicted for carboxylation of non-benzylic $\mathrm{Csp}^{3}$ carbons with $\mathrm{Rh}$ complexes, ${ }^{30-32}$ but it is in contrast to computational work on $\mathrm{Ni}$ - and $\mathrm{Pd}$-mediated carboxylations, supporting outer sphere pathways for non-benzylic $\mathrm{C}_{\mathrm{sp}}{ }^{3}$ carbons. ${ }^{32-34}$ In our computations, the $\mathrm{CO}_{2}$ insertion step is rate-limiting. The formed intermediate $4 \mathrm{a}$ has a relative energy of $-11.3 \mathrm{kcal} / \mathrm{mol}$. The final step of the catalytic cycle is the transmetalation of 4a with CsF to regenerate the active species 1 and form the product 5 (relative energy of $-15.0 \mathrm{kcal} / \mathrm{mol}$ ). No TS for this transmetalation could be located. The overall barrier of $31.9 \mathrm{kcal} / \mathrm{mol}$ for the $\mathrm{Cu}(\mathrm{I})-\mathrm{IPr}$-catalyzed carboxylation of sub1 via Path $\mathbf{A}$ is feasible at the experimental temperature of $393 \mathrm{~K}$ (based on the discussion by Baik and coworkers, ${ }^{35}$ a barrier of up to $\sim 33.2 \mathrm{kcal} / \mathrm{mol}$ should be viable). It can be noted that path 
A for $\mathrm{C}_{\mathrm{sp}}{ }^{3}$ carboxylation of the non-benzylic organoborane sub1 is similar to the reported mechanism for Cu-NHC-catalyzed $\mathrm{C}_{\mathrm{sp}}{ }^{2}$ carboxylation of arylboronate esters, ${ }^{36}$ although the involvement of $\mathrm{CSF}$ here, instead of $\mathrm{KO}^{t} \mathrm{Bu}$, provides some differences with respect to the formed intermediates and products.

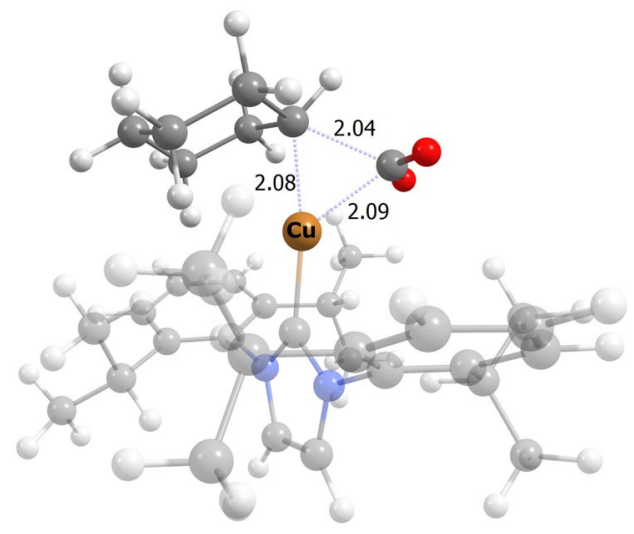

TS3a-4a(inner): $31.9 \mathrm{kcal} / \mathrm{mol}$

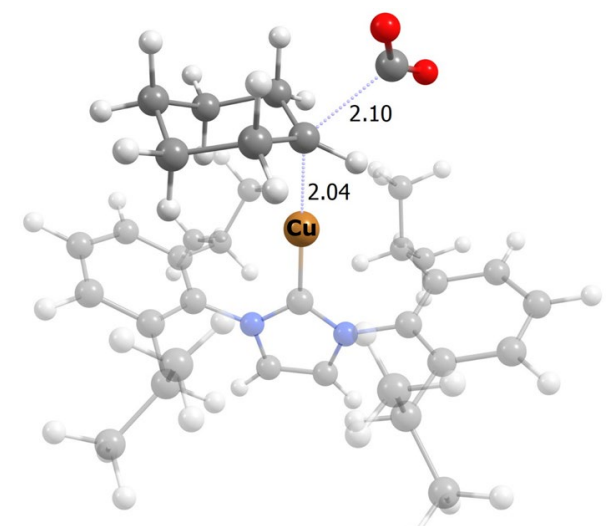

TS3a-4a(outer): $39.6 \mathrm{kcal} / \mathrm{mol}$

Figure 4. The optimized geometries of the inner and outer sphere TS for $\mathrm{CO}_{2}$ insertion (TS $3 \mathrm{a}-4 \mathrm{a}$ ) with the non-benzylic substrate sub1. Distances are in $\AA$. The NHC ligand is transparent for clarity. Color code as in Figure 3.

$\mathrm{Cu}(\mathrm{I})$-catalyzed conversion of sub2: The computed steps for the Cu-IPr-catalyzed carboxylation of the trans- $\beta$-methylstyrene-derived borane sub2 are essentially as for sub1, with the Gibbs free energy profile shown in Fig. 2. However, there are two major differences: Firstly, for the $\mathrm{CO}_{2}$

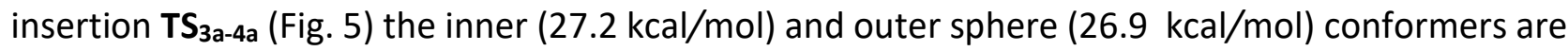
very close in energy. This indicates that for benzylic carbons, a Cu-NHC-catalyzed carboxylation may operate via both inner and outer sphere pathways. The DFT-based energies also support this conclusion (SI, Table S2 and S3). It can be noted that a recent study on Cu-NHC-mediated boracarboxylation of alkenes reported an inner sphere TS for carboxlation of a benzylic $\mathrm{C}_{\mathrm{sp}}{ }^{3}$ carbon, ${ }^{37}$ however, it is unclear if the outer sphere path was evaluated. For Rh-mediated carboxylations, we have earlier observed a very strong preference for outer sphere pathways for benzylic substrates. ${ }^{12}$ Secondly, sub2 is featuring a different resting state than sub1, which is greatly influencing the rate-limiting barrier. Our calculations predict that the complexation of sub2 with the reaction additive CsF leads to an energetically low-lying species $(\mathbf{2 b}),-9.3 \mathrm{kcal} / \mathrm{mol}$ below $\mathbf{1}$. This raises the rate-limiting barrier for $\mathrm{CO}_{2}$ insertion from $29.5 \mathrm{kcal} / \mathrm{mol}$ to $36.2 \mathrm{kcal} / \mathrm{mol}$ for sub2, which appears slightly above a feasible barrier limit at the experimental temperature ( 33.2 
$\mathrm{kcal} / \mathrm{mol}$, vide supra). It can be noted that the barrier of this step is dependent on which CsF reference state is employed in calculations. If the reference state is $\mathrm{Cs}_{4} \mathrm{~F}_{4}$ instead of $\mathrm{Cs}_{2} \mathrm{~F}_{2}$, the barrier for $\mathrm{TS}_{\mathbf{3 a}-4 a}$ is reduced from 36.2 to $33.5 \mathrm{kcal} / \mathrm{mol}$ (see SI, Table S1). Thus, there is an uncertainty in the carboxylation barrier for sub2 with Mechanism A, which is further discussed below (Table 1). For sub1, a similar CsF-substrate adduct $\mathbf{2 b}$ can also be optimized (Figure 2), but it is not lower in energy than 1, and therefore does not influence the rate-limiting barrier for sub1.

We proceeded to explore if a variant of pathway $\mathbf{A}$, with a transmetalation of the alkyl to copper occurring directly from the CsF-sub2 adduct $\mathbf{2 b}$, may provide lower barriers. However, this pathway showed unfeasible barriers of at least $47 \mathrm{kcal} / \mathrm{mol}$ for sub2 (SI, Table S4). The adduct $\mathbf{2 b}$ is thus not expected to be reactive, and it needs to dissociate prior to Cu-catalyzed transmetallation of sub2.

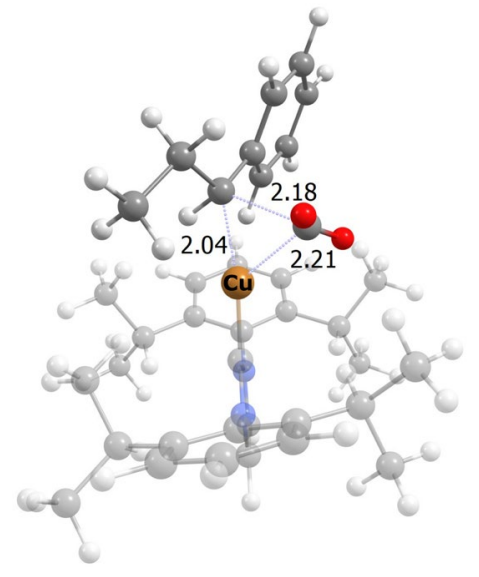

TS3a-4a(inner): $26.9 \mathrm{kcal} / \mathrm{mol}$

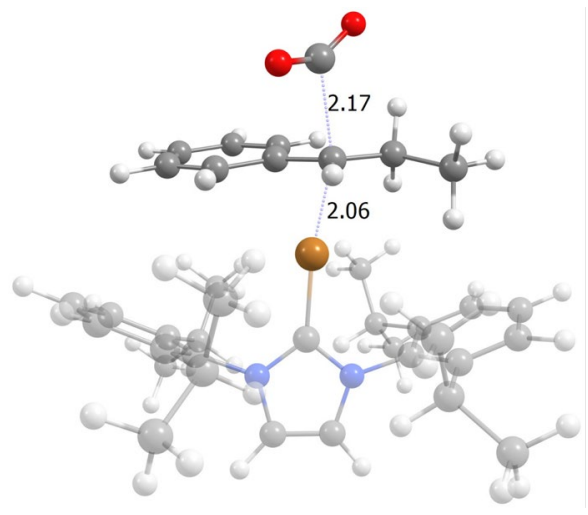

TS3a-4a(outer): $27.2 \mathrm{kcal} / \mathrm{mol}$

Figure 5. The optimized geometries for the inner- and outer sphere TS for $\mathrm{CO}_{2}$ insertion (TS $3 \mathrm{a}-4 \mathrm{a}$ ) with the benzylic substrate sub2. Distances are in $\AA$. The NHC ligand is transparent for clarity. Color code as in Figure 3.

CsF-mediated carboxylation: We recently showed that CsF can mediate the carboxylation of benzylic boranes. ${ }^{13}$ As the reaction conditions (Scheme 1) of the copper-system involves addition of three equivalents of CsF, we proceeded to explore if the additive could be the carboxylating agent. Setting out from the low-lying $\mathbf{2 b}$ adduct, a possible carboxylation pathway involving a organocaesium species $\mathbf{3 b}$ may exist, as shown in our previous work. ${ }^{13}$ This pathway will be referred to as path $\mathbf{B}$ and is shown in Scheme 3, while the corresponding computed Gibbs free energy profile is shown in Figure 6. This pathway is of no importance for sub1, for which we compute a ratelimiting barrier of $53.3 \mathrm{kcal} / \mathrm{mol}$ (Fig. 6). However, for sub2 the rate-limiting barrier computed for the Cs-mediated carboxylation path B is $37.0 \mathrm{kcal} / \mathrm{mol}$ (Fig. 6), indicating that it is equally likely to 
occur as the copper-catalyzed pathway $\mathbf{A}$ (36.2 kcal/mol, Fig. 2). It needs to be mentioned here that the TS for the rate-limiting step of path $\mathbf{B}\left(\mathbf{T S}_{\mathbf{2 b}-\mathbf{3 b}}\right)$ shows only a small imaginary frequency of $16 i$ to $35 i \mathrm{~cm}^{-1}$ with the different DFT functionals, making both its optimization and its interpretation challenging. ${ }^{38}$ However, a displacement of the TS geometry along the intrinsic reaction coordinate (IRC) supports that $\mathbf{T} \mathbf{S}_{\mathbf{2 b}-\mathbf{3 b}}$ indeed is linking $\mathbf{2} \mathbf{b}$ and $\mathbf{3 b}$, implying that the small imaginary frequency may be an inherent feature of this TS.

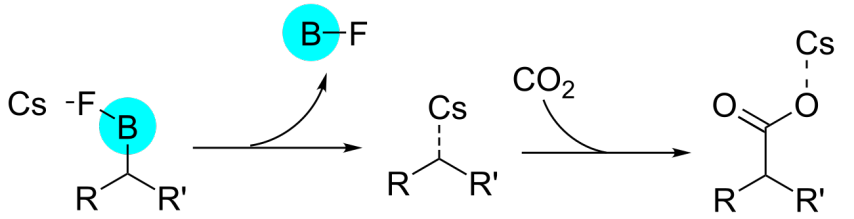

Scheme 3. Our proposed mechanism for the CsF-mediated carboxylation of benzylic organoboranes.

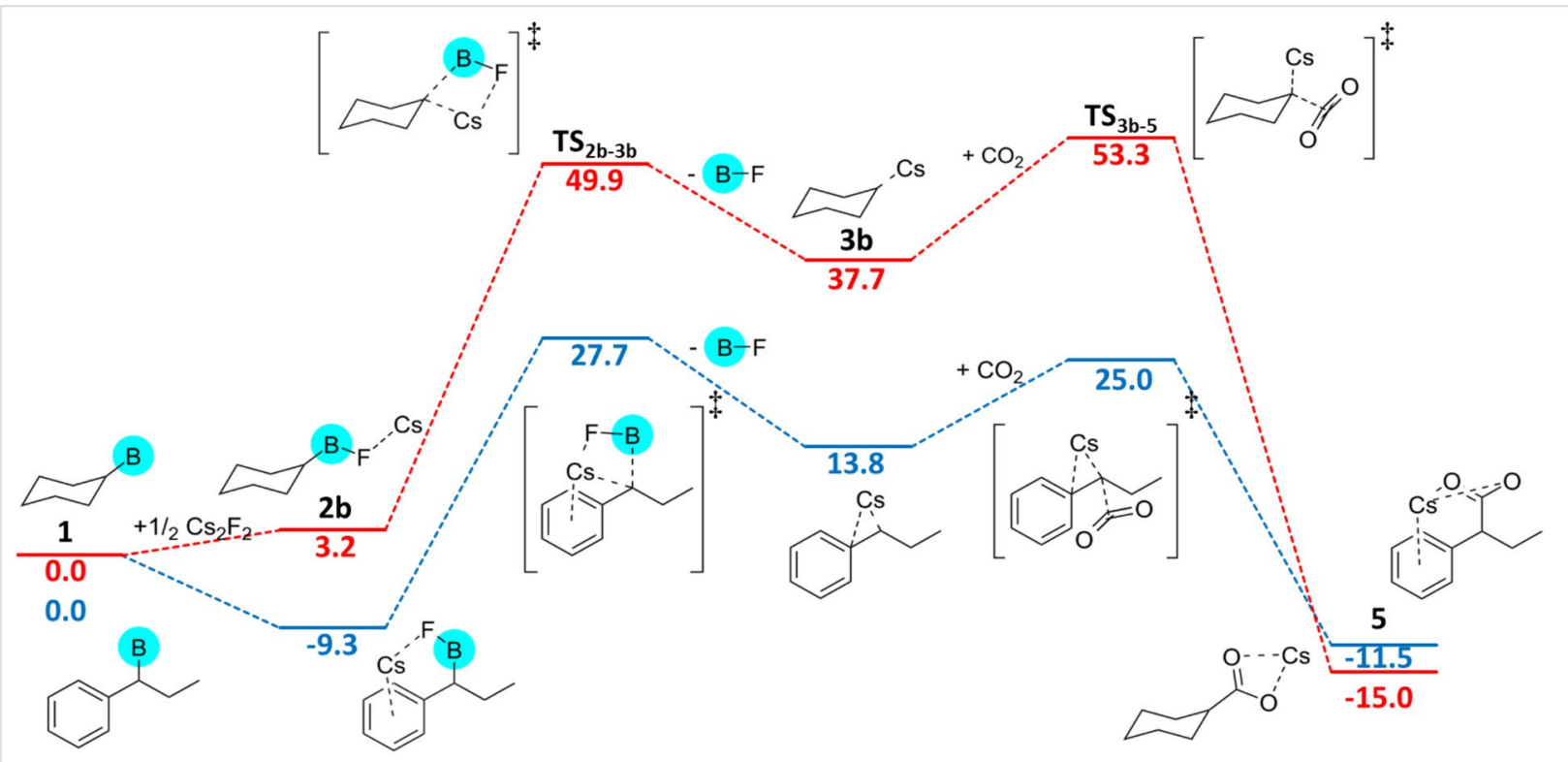

Figure 6. Computed Gibbs free energy profile $(\mathrm{kcal} / \mathrm{mol})$ of the CsF-mediated pathway B for the two substrates sub1 (red) and sub2 (blue). $B=9-B B N$

Explicit solvation effect: All calculations presented above (Fig. 2 and 6) were performed with an implicit IEFPCM model to obtain an approximate estimate of the effect of the solvent (1,4-dioxane) on the reaction geometries and energies. This is a standard approach in computational chemistry, however, it does not take into account the effect that explicit solvent may have. We here recomputed paths $\mathbf{A}$ and $\mathbf{B}$ with one explicit 1,4-dioxane molecule added as a ligand to the Cs containing species (Fig. 7). For the different computational protocols, the computed barriers (SI Table S5 and S6) are either identical or change by a maximum of $3 \mathrm{kcal} / \mathrm{mol}$ relative to the energies obtained 
with the implicit solvent model. In particular, we find that the involvement of explicit solvent does not change the proposed mechanistic preferences of sub1 (preferably path A) or sub2 (path A and B equally likely with DLPNO-CCSD(T)).

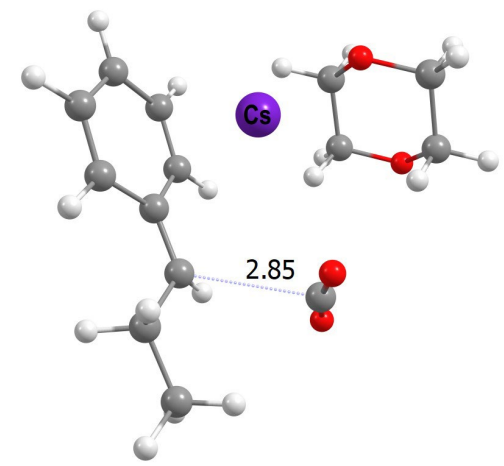

Figure 7. $\mathrm{CO}_{2}$ insertion TS (TS 3 b-5) for sub2 via path B in presence of a 1,4-dioxane molecule. Color code as in Fig. 3.

Discussion of the computed barriers: Table 1 shows the computed overall barriers at $323 \mathrm{~K}$ for the Cu-catalyzed pathway A and the CsF-mediated pathway B for sub1 and sub2 obtained with different levels of theory.

For sub1, it can be seen that the different computational protocols predict barriers that vary by up to $7.2 \mathrm{kcal} / \mathrm{mol}$. However, all methods predict that the preferred pathway is $\mathbf{A}$, which has a feasible barrier close to the discussed threshold of $33.2 \mathrm{kcal} / \mathrm{mol}$ at $323 \mathrm{~K}^{35}$ for all electronic structure methods (DLPNO-CCSD(T), $\omega B 97 X D$, PBE-D3BJ, B3LYP-D3BJ) and molecular models (with/without 1,4-dioxane) tested here. Instead, for path B, all methods and models predict a barrier above $47 \mathrm{kcal} / \mathrm{mol}$ for sub1. This strongly indicates that Cu-catalysis via path $\mathbf{A}$ is viable for sub1, whereas a CsF-mediated path $\mathbf{B}$ is excluded.

Table 1. Computed overall standard state Gibbs free energy barriers (kcal/mol, $323 \mathrm{~K}$ ) for the Cu-catalyzed pathway A and CsF-mediated pathway B obtained at different levels of theory.

\begin{tabular}{cllccc}
\hline Substrate & Method & Solvation Model & Path A & Path B & $\Delta \Delta G(A-B)$ \\
\hline sub1 & DLPNO-CCSD(T) & PCM & 31.9 & 53.3 & -21.4 \\
sub1 & DLPNO-CCSD(T) & PCM+Explicit & 31.9 & 47.7 & -15.5 \\
sub1 & wB97XD & PCM & 33.6 & 53.9 & -20.3 \\
sub1 & wB97XD & PCM+Explicit & 33.6 & 48.6 & -15.0 \\
sub1 & PBE-GD3BJ & PCM & 26.4 & $49.6^{a}$ & -23.2 \\
sub1 & B3LYP-GD3BJ & PCM & 29.9 & $50.5^{a}$ & -20.6 \\
\hline sub2 & DLPNO-CCSD(T) & PCM & 36.2 & 37.0 & -0.8 \\
sub2 & DLPNO-CCSD(T) & PCM+Explicit & 39.2 & 38.6 & 0.6 \\
sub2 & WB97XD & PCM & 40.4 & 36.4 & 4.0 \\
sub2 & wB97XD & PCM+Explicit & 40.6 & 35.6 & 5.0
\end{tabular}


$\begin{array}{llllll}\text { sub2 } & \text { PBE-GD3BJ } & \text { PCM } & 25.6 & 29.6^{\text {a }} & -4.0 \\ \text { sub2 } & \text { B3LYP-GD3BJ } & \text { PCM } & 29.8 & 28.9^{\text {a }} & 0.9\end{array}$

aEnergies based on SP calculations on the $\omega B$ 97XD structures $\left(\mathbf{T S}_{\mathbf{3 b - 5}}\right)$, as re-optimization at the given level was unsuccessful.

For sub2, the different protocols predict barriers that vary by up to $15 \mathrm{kcal} / \mathrm{mol}$ (Table 1 ). This large variation cannot be explained definitely, but one possibility is that the stability of the predicted resting state of sub2 (the low-lying CsF-substrate adduct 2b) may be described rather differently by the different methods. This state is not involved in the reactivity of sub1, where smaller energetic variations between the computational protocols are observed. The results for sub2 show a trend, where the computed barriers for both path A or B are consistently below 33 $\mathrm{kcal} / \mathrm{mol}$ with B3LYP-D3BJ or PBE-D3BJ, but consistently above $33 \mathrm{kcal} / \mathrm{mol}$ with DLPNO-CCSD(T) or $\omega B 97 X D$. Although the results indicate that both paths $\mathbf{A}$ and $\mathbf{B}$ may be equally likely to occur for sub2, a clear conclusion regarding their feasibility cannot be made. An important point in this analysis regards the expected accuracy of the computed values. Absolute barriers obtained with DFT may have an error of more than $5 \mathrm{kcal} / \mathrm{mol},{ }^{29}$ and also for DLPNO-CCSD(T), a recent benchmark showed an average deviation of more than $7 \mathrm{kcal} / \mathrm{mol}$ between experimental and computed dissociation energies, ${ }^{39}$ with the origin of the error being unknown. A conclusion on the feasibility of path $\mathbf{A}$ and $\mathbf{B}$ for sub2 thus cannot be made from computations alone. We therefore proceeded to perform experimental tests.

Experimental evaluation: A possible prediction derived from our computational work is that sub1 will only work under Cu-catalysis (path A), whereas sub2 may be converted with either Cu-catalysis (path A) or via a CsF-mediated path (path B). We tested this hypothesis by redoing the experiment in Scheme 1 with the two alkenes cyclohexene R1 (the precursor of organboborane sub1) and trans- $\beta$-methylstyrene $\mathbf{R 2}$ (the precursor of organboborane sub2), with or without Cu-IPr added to the reaction mixture (Scheme 4). For cyclohexene, the reaction gives $0 \%$ yield in the absence of copper, but $73 \%$ yield in the presence of copper. This is in excellent agreement with the computed barriers, predicting that $\mathrm{Cu}$-catalysis is essential for sub1 (Table 1). For this substrate, the results for path $\mathbf{A}$ indicate that the CSF additive only seems to be important for the transmetalation step allowing reformation of the active NHC-Cu-F species, but it should not be involved in the $\mathrm{CO}_{2}$ insertion. 
(A)

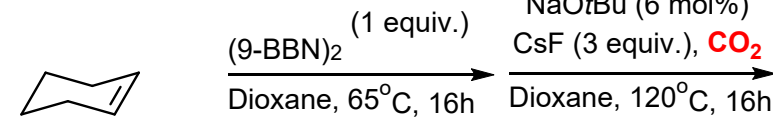

R1

(B)

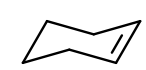

R1

(C)<smiles>[R20]#C/C=C\c1ccccc1</smiles>

(D)<smiles>C/C=C/c1ccccc1</smiles>

R2

Dioxane, $120^{\circ} \mathrm{C}, 16 \mathrm{~h}$

Cul (5 mol\%), IPrHCl (6 mol\%), Dioxane, $65^{\circ} \mathrm{C}, 16 \mathrm{~h}$ Dioxane, $120^{\circ} \mathrm{C}, 16 \mathrm{~h}$
Cul (5 mol\%),

IPrHCl (6 mol\%),

$\mathrm{NaOtBu}(6 \mathrm{~mol} \%)$

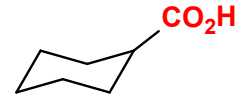

P1 73\%

$\underset{\text { Dioxane, } 65^{\circ} \mathrm{C}, 16 \mathrm{~h}}{\stackrel{(9-\mathrm{BBN}) 2}{(1 \text { equiv. })}} \stackrel{\mathrm{CsF}(3 \text { equiv. }), \mathrm{CO}_{2}}{\text { Dioxane, } 120^{\circ} \mathrm{C}, 16 \mathrm{~h}}$ $\underset{\text { Dioxane, } 65^{\circ} \mathrm{C}, 16 \mathrm{~h}}{\stackrel{(9-\mathrm{BBN}) 2}{(1 \text { equiv. })}} \stackrel{\begin{array}{c}\mathrm{NaOtBu}(6 \mathrm{~mol} \%) \\ \mathrm{CsF}(3 \text { equiv. }), \mathrm{CO}_{2}\end{array}}{\text { Dioxane, } 120^{\circ} \mathrm{C}, 16 \mathrm{~h}}$

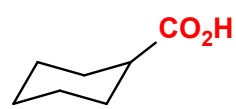

P1 $0 \%$<smiles>CCC(C(=O)O)c1ccccc1</smiles>

P2 $84 \%$<smiles>CCC(C(=O)O)c1ccccc1</smiles>

P2 91\%

Scheme 4. Experimental conditions and yield for Cu-catalyzed and CsF-mediated carboxylation of cyclohexene and trans- $\beta$-methylstyrene.

In contrast, the sub2 precursor trans- $\beta$-methylstyrene is converted to the carboxylic acid with and without copper added to the reaction mixture, providing $84 \%$ and $91 \%$ yield, respectively (Scheme 4). The higher yield in absence of copper may be attributed to a decarboxylation reaction mediated by the copper complex. ${ }^{13}$ The experimental results support the prediction that conversion of sub2 can occur in absence of copper, indicating that path B may be operative. In presence of both copper and CSF, it cannot be determined which pathway occurs for sub2, but on basis of our computations indicating similar barriers for the copper-catalyzed path $\mathbf{A}$ and the CsFmediated path B (Table 1) we predict that both occur simultaneously.

\section{Conclusions}

We have investigated the Cu-NHC-catalyzed carboxylation of cyclohexene and trans- $\beta$ methylstyrene using DFT and DLPNO-CCSD(T). Several interesting conclusions can be drawn. Firstly, a main conclusion is that non-benzylic organoboranes such as sub1 require copper for successful 
$\mathrm{CO}_{2}$ insertion, whereas for the benzylic organoborane sub2, the copper-catalyzed reaction and the CsF-mediated carboxylation path appear equally accessible. This behavior is consisted for different computational protocols and models. Our experimental testing verified the need for copper for sub1, but showed good carboxylation of the benzylic carbon in sub2 in absence of copper.

Secondly, an analysis of the involved transition states shows that non-benzylic $\mathrm{C}_{\mathrm{sp}}{ }^{3}$ substrates prefer an inner sphere carboxylation (where $\mathrm{CO}_{2}$ exhibits interactions with the metal center), whereas the benzylic $\mathrm{C}_{\mathrm{sp}}{ }^{3}$ species can proceed along both inner and outer sphere routes for $\mathrm{CO}_{2}$ insertion. A clear consensus regarding inner versus outer sphere mechanism for $\mathrm{C}-\mathrm{CO}_{2}$ bond formation is currently not available from the literature. Both inner and outer have been predicted for metal-coordinated $\mathrm{C}_{\mathrm{sp}}{ }^{3}$ carbons, ${ }^{12,30-34,37}$ although sometimes only one alternative appears to have been studied computationally. The intimate details of the $\mathrm{CO}_{2}$ insertion step are of particular interest for developing enantioselective carboxylations, where the inner and outer pathways would give opposite configurations. Our conclusions that both $\mathrm{CO}_{2}$ insertion modes may occur simultaneously in Cu-NHC-based carboxylations of benzylic carbons indicate that attempts to develop an asymmetric variant of this type of reactions are unlikely to succeed. More investigations regarding the effect of the metal catalyst and substrate on the preferred $\mathrm{CO}_{2}$ insertion mode are in progress in our laboratory.

\section{Supporting Information.}

Cartesian coordinates in a format that allows visualization with e.g. Mercury (.xyz file). Additional computational results, experimental procedures, data for compound characterisation and NMR spectra (.pdf file). This material is available free of charge via the Internet at http://pubs.acs.org.

\section{Notes}

The authors declare no competing financial interests.

\section{Acknowledgements}

This work has been supported by the Research Council of Norway through a Centre of Excellence Grant (No. 262695), by the Troms $\varnothing$ Research Foundation (No. TFS2016KHH), by Notur - The Norwegian Metacenter for 
Computational Science through grants of computer time (No. nn9330k and nn4654k), and by NordForsk (No. 85378 ) and the members of the Nordic Consortium for $\mathrm{CO}_{2}$ Conversion $\left(\mathrm{NordCO}_{2}\right)$.

\section{References}

1. Otto, A.; Grube, T.; Schiebahn, S.; Stolten, D., Closing the loop: captured $\mathrm{CO}_{2}$ as a feedstock in the chemical industry. Energy Environ. Sci. 2015, 8, 3283-3297.

2. Dai, W.-L.; Luo, S.-L.; Yin, S.-F.; Au, C.-T., The direct transformation of carbon dioxide to organic carbonates over heterogeneous catalysts. Appl. Catal. A Gen. 2009, 366, 2-12.

3. Ma, J.; Sun, N.; Zhang, X.; Zhao, N.; Xiao, F.; Wei, W.; Sun, Y., A short review of catalysis for $\mathrm{CO}_{2}$ conversion. Catal. Today 2009, 148, 221-231.

4. Liu, Q.; Wu, L.; Jackstell, R.; Beller, M., Using carbon dioxide as a building block in organic synthesis. Nat. Commun. 2015, 6, 5933.

5. Dabral, S.; Schaub, T., The Use of Carbon Dioxide $\left(\mathrm{CO}_{2}\right)$ as a Building Block in Organic Synthesis from an Industrial Perspective. Adv. Synth. Catal. 2019, 361, 223-246.

6. Aresta, M.; Dibenedetto, A.; Angelini, A., Catalysis for the valorization of exhaust carbon: from $\mathrm{CO}_{2}$ to chemicals, materials, and fuels. Technological use of $\mathrm{CO}_{2}$. Chem. Rev. 2014, 114, 1709-42.

7. Aresta, M.; Dibenedetto, A.; Quaranta, E., State of the art and perspectives in catalytic processes for $\mathrm{CO}_{2}$ conversion into chemicals and fuels: The distinctive contribution of chemical catalysis and biotechnology. $J$. Catal. 2016, 343, 2-45.

8. Shaikh, R. R.; Pornpraprom, S.; D'Elia, V., Catalytic Strategies for the Cycloaddition of Pure, Diluted, and Waste $\mathrm{CO}_{2}$ to Epoxides under Ambient Conditions. ACS Catal. 2017, 8, 419-450.

9. Ohishi, T.; Zhang, L.; Nishiura, M.; Hou, Z., Carboxylation of alkylboranes by N-heterocyclic carbene copper catalysts: synthesis of carboxylic acids from terminal alkenes and carbon dioxide. Angew. Chem. Int. Ed. 2011, 50, 8114-7.

10. Ohmiya, H.; Tanabe, M.; Sawamura, M., Copper-catalyzed carboxylation of alkylboranes with carbon dioxide: formal reductive carboxylation of terminal alkenes. Org. Lett. 2011, 13, 1086-8.

11. Juhl, M.; Laursen, S. L. R.; Huang, Y.; Nielsen, D. U.; Daasbjerg, K.; Skrydstrup, T., Copper-Catalyzed Carboxylation of Hydroborated Disubstituted Alkenes and Terminal Alkynes with Cesium Fluoride. ACS Catal. 2017, 7, 1392-1396.

12. Pavlovic, L.; Vaitla, J.; Bayer, A.; Hopmann, K. H., Rhodium-Catalyzed Hydrocarboxylation: Mechanistic Analysis Reveals Unusual Transition State for Carbon-Carbon Bond Formation. Organometallics 2018, 37, 941948.

13. Gevorgyan, A.; Obst, M. F.; Guttormsen, Y.; Maseras, F.; Hopmann, K. H.; Bayer, A., Caesium FluorideMediated Hydrocarboxylation of Alkenes and Allenes: Scope and Mechanistic Insights. Chem. Sci. 2019, 10, 10072-10078.

14. Chai, J. D.; Head-Gordon, M., Long-range corrected hybrid density functionals with damped atom-atom dispersion corrections. Phys. Chem. Chem. Phys. 2008, 10, 6615-6620.

15. Goerigk, L.; Hansen, A.; Bauer, C.; Ehrlich, S.; Najibi, A.; Grimme, S., A look at the density functional theory zoo with the advanced GMTKN55 database for general main group thermochemistry, kinetics and noncovalent interactions. Phys. Chem. Chem. Phys. 2017, 19, 32184-32215.

16. Mardirossian, N.; Head-Gordon, M., Thirty years of density functional theory in computational chemistry: an overview and extensive assessment of 200 density functionals. Mol. Phys. 2017, 115, 2315-2372.

17. Perdew, J. P.; Burke, K.; Ernzerhof, M., Generalized Gradient Approximation Made Simple. Phys. Rev. Lett. 1996, 77, 3865-3868.

18. Perdew, J. P.; Burke, K.; Ernzerhof, M., Generalized Gradient Approximation Made Simple [Phys. Rev. Lett. 77, 3865 (1996)]. Phys. Rev. Lett. 1997, 78, 1396-1396.

19. Becke, A. D., Density-functional thermochemistry. III. The role of exact exchange. J. Chem. Phys. 1993, 98, 5648-5652.

20. Grimme, S.; Ehrlich, S.; Goerigk, L., Effect of the damping function in dispersion corrected density functional theory. J. Comput. Chem. 2011, 32, 1456-65.

21. Frisch, M. J.; Trucks, G. W.; Schlegel, H. B.; Scuseria, G. E.; Robb, M. A.; Cheeseman, J. R.; Scalmani, G.; 
Barone, V.; Petersson, G. A.; Nakatsuji, H.; Li, X.; Caricato, M.; Marenich, A. V.; Bloino, J.; Janesko, B. G.; Gomperts, R.; Mennucci, B.; Hratchian, H. P.; Ortiz, J. V.; Izmaylov, A. F.; Sonnenberg, J. L.; Williams; Ding, F.; Lipparini, F.; Egidi, F.; Goings, J.; Peng, B.; Petrone, A.; Henderson, T.; Ranasinghe, D.; Zakrzewski, V. G.; Gao, J.; Rega, N.; Zheng, G.; Liang, W.; Hada, M.; Ehara, M.; Toyota, K.; Fukuda, R.; Hasegawa, J.; Ishida, M.; Nakajima, T.; Honda, Y.; Kitao, O.; Nakai, H.; Vreven, T.; Throssell, K.; Montgomery Jr., J. A.; Peralta, J. E.; Ogliaro, F.; Bearpark, M. J.; Heyd, J. J.; Brothers, E. N.; Kudin, K. N.; Staroverov, V. N.; Keith, T. A.; Kobayashi, R.; Normand, J.; Raghavachari, K.; Rendell, A. P.; Burant, J. C.; lyengar, S. S.; Tomasi, J.; Cossi, M.; Millam, J. M.; Klene, M.; Adamo, C.; Cammi, R.; Ochterski, J. W.; Martin, R. L.; Morokuma, K.; Farkas, O.; Foresman, J. B.; Fox, D. J. Gaussian 16 Rev. B.01, Wallingford, CT, 2016.

22. Riplinger, C.; Neese, F., An efficient and near linear scaling pair natural orbital based local coupled cluster method. J. Chem. Phys. 2013, 138, 034106.

23. Riplinger, C.; Sandhoefer, B.; Hansen, A.; Neese, F., Natural triple excitations in local coupled cluster calculations with pair natural orbitals. J. Chem. Phys. 2013, 139, 134101.

24. Sparta, M.; Neese, F., Chemical applications carried out by local pair natural orbital based coupled-cluster methods. Chem. Soc. Rev. 2014, 43, 5032-41.

25. Liakos, D. G.; Sparta, M.; Kesharwani, M. K.; Martin, J. M. L.; Neese, F., Exploring the Accuracy Limits of Local Pair Natural Orbital Coupled-Cluster Theory. J. Chem. Theory Comput. 2015, 11, 1525-1539.

26. Minenkov, Y.; Chermak, E.; Cavallo, L., Accuracy of DLPNO-CCSD(T) method for noncovalent bond dissociation enthalpies from coinage metal cation complexes. J. Chem. Theory Comput. 2015, 11, 4664-76.

27. Neese, F.; Wennmohs, F.; Hansen, A.; Becker, U., Efficient, approximate and parallel Hartree-Fock and hybrid DFT calculations. A 'chain-of-spheres' algorithm for the Hartree-Fock exchange. Chem. Phys. 2009, 356, 98109.

28. Neese, F., The ORCA program system. WIREs Comput. Mol. Sci. 2012, 2, 73-78.

29. Hopmann, K. H., How Accurate is DFT for Iridium-Mediated Chemistry? Organometallics 2016, 35, 3795-3807.

30. Ostapowicz, T. G.; Hölscher, M.; Leitner, W., Catalytic Hydrocarboxylation of Olefins with $\mathrm{CO}_{2}$ and $\mathrm{H}_{2}-$ a DFT Computational Analysis. Eur. J. Inorg. Chem. 2012, 2012, 5632-5641.

31. Ostapowicz, T. G.; Hölscher, M.; Leitner, W., $\mathrm{CO}_{2}$ insertion into metal-carbon bonds: a computational study of $\mathrm{Rh}(\mathrm{I})$ pincer complexes. Chem. Eur. J. 2011, 17, 10329-38.

32. Schmeier, T. J.; Hazari, N.; Incarvito, C. D.; Raskatov, J. A., Exploring the reactions of $\mathrm{CO}_{2}$ with $\mathrm{PCP}$ supported nickel complexes. Chem. Commun. 2011, 47, 1824-6.

33. Lau, K.-C.; Petro, B. J.; Bontemps, S.; Jordan, R. F., Comparative Reactivity of Zr-and Pd-Alkyl Complexes with Carbon Dioxide. Organometallics 2013, 32, 6895-6898.

34. Johnson, M. T.; Johansson, R.; Kondrashov, M. V.; Steyl, G.; Ahlquist, M. r. S. G.; Roodt, A.; Wendt, O. F., Mechanisms of the $\mathrm{CO}_{2}$ Insertion into (PCP) Palladium Allyl and Methyl $\sigma$-Bonds. A Kinetic and Computational Study. Organometallics 2010, 29, 3521-3529.

35. Ryu, H.; Park, J.; Kim, H. K.; Park, J. Y.; Kim, S.-T.; Baik, M.-H., Pitfalls in Computational Modeling of Chemical Reactions and How To Avoid Them. Organometallics 2018, 37, 3228-3239.

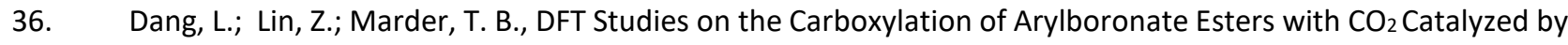
Copper(I) Complexes. Organometallics 2010, 29, 917-927.

37. Lv, X.; Wu, Y.-B.; Lu, G., Computational exploration of ligand effects in copper-catalyzed boracarboxylation of styrene with $\mathrm{CO}_{2}$. Catal. Sci. Technol. 2017, 7, 5049-5054.

38. The imaginary frequency is inversely proportional to the square root of the reduced mass of the vibrating atoms. If many atoms are displaced during the TS, the imaginary frequency will necessarily be small. The obtained imaginary frequencies for $\mathbf{T S}_{\mathbf{2 b}-3 \mathbf{b}}$ for sub1 and sub2 are respectively $19 i$ and $16 i \mathrm{~cm}^{-1}$ for $\omega \mathrm{B} 97 \mathrm{XD}, 25 i$ $\mathrm{cm}^{-1}$ and $23 i \mathrm{~cm}^{-1}$ for B3LYP-D3, and $35 i$ and $13 i \mathrm{~cm}^{-1}$ for PBE-D3BJ.

39. Husch, T.; Freitag, L.; Reiher, M., Calculation of Ligand Dissociation Energies in Large Transition-Metal Complexes. J. Chem. Theory Comput. 2018, 14, 2456-2468. 


\section{TOC graphic}
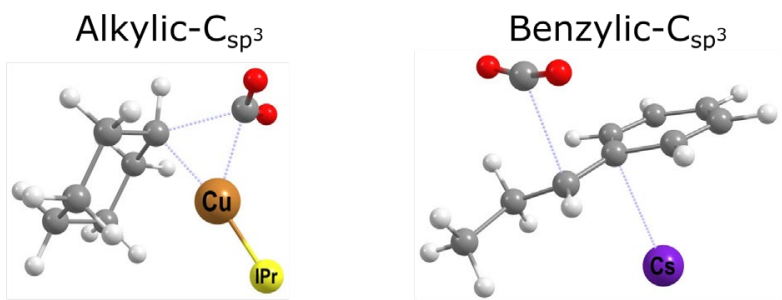

- Cu-mediated

- Cu- \& CsF-mediated

- Metal- $\mathrm{CO}_{2}$ interaction required

- Metal- $\mathrm{CO}_{2}$ interaction not required 